\title{
MULTIFRACTAL ANALYSIS FOR BIRKHOFF AVERAGES ON LALLEY-GATZOURAS REPELLERS
}

\author{
HENRY WJ REEVE
}

\begin{abstract}
We consider the multifractal analysis for Birkhoff averages of continuous potentials on a class of non-conformal repellers corresponding to the self-affine limit sets studied by Lalley and Gatzouras. A conditional variational principle is given for the Hausdorff dimension of the set of points for which the Birkhoff averages converge to a given value. This extends a result of Barral and Mensi to certain non-conformal maps with a measure dependent Lyapunov exponent.
\end{abstract}

\section{INTRODUCTION AND STATEMENT OF RESUlts}

In this paper we consider the multifractal analysis of Birkhoff averages. Let $\Lambda$ be a repeller for a planar map $T: \mathbb{R}^{2} \rightarrow \mathbb{R}^{2}$. Given a continuous potential $\varphi: \Lambda \rightarrow \mathbb{R}$ and $\alpha \in \mathbb{R}$ we are interested in the set of those points in the repeller for which the Birkhoff average converges to $\alpha$

$$
\Lambda_{\alpha}^{\varphi}:=\left\{x \in \Lambda: \lim _{n \rightarrow \infty} \frac{1}{n} \sum_{k=0}^{n-1} \varphi\left(T^{k}(x)\right)=\alpha\right\} .
$$

In particular we would like to understand how the Hausdorff dimension $\operatorname{dim}_{\mathcal{H}}$ of $\Lambda_{\alpha}^{\varphi}$ varies as a function of $\alpha$,

$$
\alpha \mapsto \operatorname{dim}_{\mathcal{H}} \Lambda_{\alpha}^{\varphi} .
$$

When $T$ is conformal and hyperbolic the function $\alpha \mapsto \operatorname{dim}_{\mathcal{H}} \Lambda_{\alpha}^{\varphi}$ is well understood (see Pesin and Weiss [18, Fan Feng and Wu [7], Barriera and Saussol [4] and Olsen [15] for increasingly general results). However, in the non-conformal setting much less is known. Jordan and Simon [11] gave a variational formula for $\operatorname{dim}_{\mathcal{H}} \Lambda_{\alpha}^{\varphi}$ for typical members of families of piecewise diagonal maps. Barral and Mensi [2] and Barral and Feng [1] give a precise formula for $\operatorname{dim}_{\mathcal{H}} \Lambda_{\alpha}^{\varphi}$ in the setting of Bedford [5] and McMullen [13].

The author would like to thank his supervisor Thomas Jordan for all his help in preparing this paper. This project was started in the Instytut Matematyczny PAN and I would like to thank the Institute, especially Michał Rams and Feliks Przytycki, for their kind hospitality. I would also like to thank the Engineering and Physical Sciences Research Council and the Conformal Structures and Dynamics network for their financial support. 

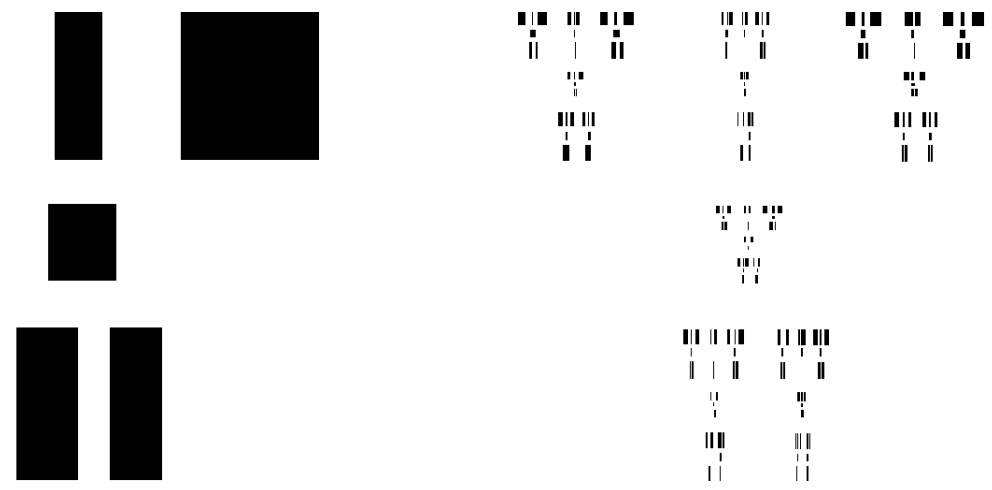

FiguRE 1. A representation of a Lalley-Gatzouras system (left) and the corresponding limit set (right).

We shall prove a conditional variational principle for $\operatorname{dim}_{\mathcal{H}} \Lambda_{\alpha}^{\varphi}$ for a more general class of piecewise affine maps $T: \mathbb{R}^{2} \rightarrow \mathbb{R}^{2}$ with repellers $\Lambda$ corresponding to the self-affine limit sets studied by Lalley and Gatzouras in [8].

Definition 1.1 (Lalley-Gatzouras Systems). Suppose we have some index set $\mathcal{D}=\left\{(i, j): 1 \leq i \leq p\right.$ and $\left.1 \leq j \leq m_{i}\right\}$ and for each $(i, j) \in \mathcal{D}$ we define an affine contraction of the form

$$
S_{i j}(x)=\left(\begin{array}{ll}
a_{i j} & 0 \\
0 & b_{j}
\end{array}\right) x+\left(\begin{array}{c}
c_{i j} \\
d_{i}
\end{array}\right) \text { for } x \in[0,1]^{2}
$$

where $a_{i j}, b_{i}, c_{i j}, d_{i}$ are fixed members of $[0,1]$, with $b_{i}$ and $d_{i}$ depending only on $i$. Suppose that for each $(i, j) \in \mathcal{D}$ we have $0<a_{i j} \leq b_{i}<1$. We stipulate that $0 \leq d_{1} \leq d_{2} \leq \cdots \leq d_{p}<1$ with $d_{i+1}-d_{i} \geq b_{i}$ and $b_{p}+d_{p} \leq 1$ and for each $i, 0 \leq c_{i 1} \leq c_{i 2} \leq \cdots \leq c_{i m_{i}}<1$ with $c_{i(j+1)}-c_{i j} \geq a_{i j}$ and $a_{i m_{i}}+c_{i m_{i}} \leq 1$. We shall refer to a family of affine maps $\left(S_{i j}\right)_{(i, j) \in \mathcal{D}}$, formed in this way, as a Lalley-Gatzouras system.

Let $\Sigma:=\mathcal{D}^{\mathbb{N}}$ and $\Sigma_{v}:=\{1, \cdots, p\}^{\mathbb{N}}$ be full shift spaces with corresponding left shift operators denoted by $\sigma: \Sigma \rightarrow \Sigma$ and $\sigma_{v}: \Sigma_{v} \rightarrow \Sigma_{v}$, respectively. Given $\omega \in \Sigma$ and $n \in \mathbb{N}$ we let $\omega \mid n \in \mathcal{D}$ denote the finite string consisting of the first $n$ terms of $\omega$. We define $\pi: \Sigma \rightarrow \Sigma_{v}$ by $\pi:\left(\left(i_{n}, j_{n}\right)\right)_{n \in \mathbb{N}} \mapsto\left(i_{n}\right)_{n \in \mathbb{N}}$. Given $n \in \mathbb{N}$ we let $\mathcal{M}_{\sigma^{n}}(\Sigma)$ denote the set of Borel probability measures supported on $\Sigma$ which are invariant under $\sigma^{n}$ and $\mathcal{B}_{\sigma^{n}}(\Sigma)$ the set of Bernoulli measures with respect to $\sigma^{n}$. Similarly, we let $\mathcal{M}_{\sigma_{v}^{n}}\left(\Sigma_{v}\right)$ denote the set of $\sigma_{v}^{n}$-invariant measures and $\mathcal{B}_{\sigma_{v}^{n}}\left(\Sigma_{v}\right)$ the set of $\sigma_{v}^{n}$-Bernoulli measures. Note that if $\mu \in \mathcal{M}_{\sigma^{n}}(\Sigma)$ then $\pi(\mu):=\mu \circ \pi^{-1} \in \mathcal{M}_{\sigma_{v}^{n}}\left(\Sigma_{v}\right)$ and if $\mu \in \mathcal{B}_{\sigma^{n}}(\Sigma)$ then $\pi(\mu) \in \mathcal{B}_{\sigma_{v}^{n}}\left(\Sigma_{v}\right)$. Given $n \in \mathbb{N}$ and $\mu \in \mathcal{M}_{\sigma^{n}}(\Sigma)$ we define corresponding Lyapunov exponents by

$$
\lambda\left(\mu, \sigma^{n}\right):=-\int \log a_{\omega_{1}} \cdots a_{\omega_{n}} d \mu(\omega)
$$


and

$$
\lambda^{v}\left(\mu, \sigma^{n}\right):=-\int \log b_{i_{1}} \cdots b_{i_{n}} d \mu(\omega) .
$$

We also let $h\left(\mu, \sigma^{n}\right)$ denote the Kolmogorov-Sinai entropy of $\mu$ with respect to $\sigma^{n}$ and $h^{v}\left(\mu, \sigma^{n}\right)$ the Kolmogorov-Sinai entropy of $\pi(\mu)$ with respect to $\sigma_{v}^{n}$.

Given $n \in \mathbb{N}$ and $\mu \in \mathcal{M}_{\sigma^{n}}(\Sigma)$ we define

$$
D_{L Y}^{n}(\mu)=\frac{h\left(\mu, \sigma^{n}\right)}{\lambda\left(\mu, \sigma^{n}\right)}+\left(\frac{1}{\lambda^{v}\left(\mu, \sigma^{n}\right)}-\frac{1}{\lambda\left(\mu, \sigma^{n}\right)}\right) h^{v}\left(\mu, \sigma^{n}\right)
$$

cf. Ledrappier and Young [12] Corollary D. We write $D_{L Y}^{1}(\mu)$ as $D_{L Y}(\mu)$. Let $C(\Sigma)$ denote the set of continuous potentials $\varphi: \Sigma \rightarrow \mathbb{R}$. Given $\varphi \in$ $C(\Sigma)$ and $\alpha \in \mathbb{R}$ we let

$$
\Sigma_{\alpha}^{\varphi}:=\left\{\omega \in \Sigma: \lim _{n \rightarrow \infty} \frac{1}{n} \sum_{l=0}^{n-1} \varphi\left(\sigma^{l}(\omega)\right)=\alpha\right\} .
$$

Let $\alpha_{\min }(\varphi):=\inf \left\{\int \varphi d \mu: \mu \in \mathcal{M}_{\sigma}(\Sigma)\right\}$ and $\alpha_{\max }(\varphi):=\sup \left\{\int \varphi d \mu: \mu \in \mathcal{M}_{\sigma}(\Sigma)\right\}$. It is easy to check that $\left[\alpha_{\min }(\varphi), \alpha_{\max }(\varphi)\right]=\left\{\alpha \in \mathbb{R}: \Sigma_{\alpha}^{\varphi} \neq \emptyset\right\}$ (apply [19] Theorems 1.14 and 6.9). Given a potential $\varphi: \Sigma \rightarrow \mathbb{R}$, we define for each $k \in \mathbb{N}$ the $k$ th average potential $A_{k}(\varphi): \Sigma \rightarrow \mathbb{R}$ by $A_{k}(\varphi):=\frac{1}{k} \sum_{l=0}^{k-1} \varphi \circ \sigma^{l}$ and the $k$ variance $\operatorname{var}_{k}(\varphi):=\sup \left\{|\varphi(\omega)-\varphi(\tau)|: \omega_{l}=\tau_{l}\right.$ for $\left.l=1, \cdots, k\right\}$. Let $\chi^{h}:[0,1]^{2} \rightarrow[0,1]$ denote the horizontal projection given by $\left(x_{1}, x_{2}\right) \mapsto$ $x_{1}$ and $\chi^{v}:[0,1]^{2} \rightarrow[0,1]$ the vertical projection $\left(x_{1}, x_{2}\right) \mapsto x_{2}$. For each $(i, j) \in \mathcal{D}$ we let $f_{i j}$ denote the affine map $x \mapsto a_{i j} x+c_{i j}$ and $g_{i}$ denote the affine map $x \mapsto b_{i} x+d_{i}$. It follows that $f_{i j} \circ \chi^{h}=\chi^{h} \circ S_{i j}$ and $g_{i} \circ \chi^{v}=\chi^{v} \circ S_{i j}$. Given a finite string $\eta=\eta_{1} \cdots \eta_{n} \in \mathcal{D}^{n}$ we let $S_{\eta}:=S_{\eta_{1}} \circ \cdots \circ S_{\eta_{n}}$ and $f_{\eta}:=f_{\eta_{1}} \circ \cdots \circ f_{\eta_{n}}$. Similarly given $\zeta=\zeta_{1} \cdots \zeta_{n} \in\{1, \cdots, p\}^{n}$ we let $g_{\zeta}:=g_{\zeta_{1}} \circ \cdots \circ g_{\zeta_{n}}$. There is a natural projection $\Pi: \Sigma \rightarrow \mathbb{R}^{2}$ given by

$$
\Pi(\omega)=\lim _{n \rightarrow \infty} S_{\omega \mid n}\left([0,1]^{2}\right) .
$$

Define $\Lambda:=\Pi(\Sigma)$ and for each $\varphi \in C(\Sigma)$ and $\alpha \in \mathbb{R}$ we let $\Lambda_{\alpha}^{\varphi}:=$ $\Pi\left(\Sigma_{\alpha}^{\varphi}\right)$. Note that $\Lambda$ is the unique non-empty compact set satisfying $\Lambda=$ $\bigcup_{(i, j) \in \mathcal{D}} S_{i j}(\Lambda)$. It was shown by Lalley and Gatzouras in [8] that,

Theorem 1.1. (Lalley and Gatzouras, 1992)

$$
\operatorname{dim}_{\mathcal{H}} \Lambda=\sup \left\{D_{L Y}(\mu): \mu \in \mathcal{B}_{\sigma}(\Sigma)\right\} .
$$

The central purpose of this paper is to prove Theorem 1 .

Theorem 1. Suppose $\varphi \in C(\Sigma)$. Then for all $\alpha \in\left[\alpha_{\min }(\varphi), \alpha_{\max }(\varphi)\right]$ we have

$$
\operatorname{dim}_{\mathcal{H}} \Lambda_{\alpha}^{\varphi}=\sup \left\{D_{L Y}(\mu): \mu \in \mathcal{M}_{\sigma}(\Sigma), \int \varphi d \mu=\alpha\right\} .
$$

In particular $\alpha \mapsto \operatorname{dim}_{\mathcal{H}} \Lambda_{\alpha}^{\varphi}$ is continuous on $\left[\alpha_{\min }(\varphi), \alpha_{\max }(\varphi)\right]$. 
Corresponding to each Lalley-Gatzouras IFS satisfying $S_{i_{1} j_{1}}\left([0,1]^{2}\right) \cap$ $S_{i_{2} j_{2}}\left([0,1]^{2}\right)=\emptyset$ for $\left(i_{1}, j_{1}\right) \neq\left(i_{2}, j_{2}\right) \in \mathcal{D}$ there is an associated piecewise affine planar map $T: \mathbb{R}^{2} \rightarrow \mathbb{R}^{2}$. $T$ is the unique orientation preserving piecewise affine map which sends each rectangle $R_{i j}:=\left[c_{i j}, c_{i j}+a_{i j}\right] \times\left[d_{i}, d_{i}+b_{i}\right]$ to $[0,1]^{2}$ and leaves the rest of the plane fixed. The set

$$
\Lambda=\left\{x \in \mathbb{R}^{2}: T^{n}(x) \in \bigcup_{(i, j) \in \mathcal{D}} R_{i j} \text { for all } n \geq 0\right\}
$$

is a repeller for $T$, known as a Lalley-Gatzouras repeller. The dynamical interest in Theorem 1 is that it allows us to give the multifractal analysis for Birkhoff averages (see 1.2 above) for maps $T: \mathbb{R}^{2} \rightarrow \mathbb{R}^{2}$ of this form. Note that the special case of Theorem 1 in which each of the maps $S_{i j}$ is a similarity may be deduced from Olsen [15] Theorem 1. Moreover, the special case in which there exists constants $a, b$ for which $a_{i j}=a$ and $b_{i}=b$ for all $(i, j) \in \mathcal{D}$ was solved by Barral and Mensi in [2] using a weighted version of the thermodynamic formalism. However, when we are in the non-conformal setting with measure dependent Lyapunov exponents the thermodynamic formalism does not apply and a different approach is required. For the lower bound, we combine ideas from Lalley and Gatzouras [8 and Gelfert and Rams [9. For the upper bound, we begin by adapting a technique from Barański [3] to prove the result for locally constant potentials before applying an approximation argument to obtain the result in full generality. The paper is structured as follows. In section 2 we recall the notion of an approximate square, demonstrating how they may be used to give dimension estimates for projections of subsets of the symbolic space. In section 3 we prove the lower bound and in section 4 we prove the upper bound. We conclude with some remarks and an open question.

\section{Dimension Lemmas}

An estimate for Hausdorff dimension is obtained by finding optimal coverings. In the conformal setting it ordinarily suffices to consider families of projections of cylinder sets. However, in the non-conformal setting the geometric distortion resulting from a difference in expansion between the strong and the weak unstable foliation means that coverings of this form will be highly non-optimal. Instead we follow McMullen [13] and Lalley and Gatzouras [8] in using approximate squares for this purpose.

We define for each $\omega \in \Sigma$ and $n \in \mathbb{N}$

$$
L_{n}(\omega):=\min \left\{l \geq 1: \prod_{\nu=1}^{l} a_{i_{\nu} j_{\nu}} \leq \prod_{\nu=1}^{n} b_{i_{\nu}}\right\} .
$$

Note that this implies

$$
a_{\min }<\frac{\prod_{\nu=1}^{L_{n}(\omega)} a_{i_{\nu} j_{\nu}}}{\prod_{\nu=1}^{n} b_{i_{\nu}}} \leq 1 .
$$


Given $\left(\omega_{\nu}\right)_{\nu=1}^{n}=\left(\left(i_{\nu}, j_{\nu}\right)\right)_{\nu=1}^{n} \in \mathcal{D}^{n}$ we let

$$
\left[\omega_{1} \cdots \omega_{n}\right]:=\left\{\omega^{\prime} \in \Sigma: \omega_{\nu}^{\prime}=\omega_{\nu} \text { for } \nu=1, \cdots, n\right\}
$$

and

$$
\left[i_{1} \cdots i_{n}\right]:=\left\{\omega^{\prime} \in \Sigma: i_{\nu}^{\prime}=i_{\nu} \text { for } \nu=1, \cdots, n\right\} .
$$

Given $\omega=\left(\left(i_{\nu}, j_{\nu}\right)\right)_{\nu=1}^{\infty} \in \Sigma$ we let $B_{n}(\omega)$ denote the $n$th approximate symbolic square,

$$
B_{n}(\omega):=\left[\omega_{1} \cdots \omega_{L_{n}(\omega)}\right] \cap \sigma^{-L_{n}(\omega)}\left[i_{L_{n}(\omega)+1} \cdots i_{n}\right] .
$$

We let $\Delta_{n}(\omega)$ denote the approximate square corresponding to $B_{n}(\omega)$, defined by

$$
\Delta_{n}(\omega):=f_{\omega \mid L_{n}(\omega)}([0,1]) \times g_{\mathbf{i} \mid n}([0,1]) .
$$

Note that for each $\omega \in \Sigma$ and $n \in \mathbb{N}, \Pi\left(B_{n}(\omega)\right) \subseteq \Delta_{n}(\omega)$ and for all $\omega^{\prime} \notin B_{n}(\omega), \operatorname{int}\left(\Delta_{n}(\omega)\right) \cap \operatorname{int}\left(\Delta_{n}\left(\omega^{\prime}\right)\right)=\emptyset$.

We say that the digit set $\mathcal{D}$ is two-dimensional if there exists $\left(i_{1}, j_{1}\right),\left(i_{2}, j_{2}\right) \in$ $\mathcal{D}$ with $i_{1}=i_{2}$ and $j_{1} \neq j_{2}$ and there exists $\left(i_{3}, j_{3}\right),\left(i_{4}, j_{4}\right) \in \mathcal{D}$ with $i_{3} \neq i_{4}$. Define for each $d \in \mathcal{D}$

$$
R_{n}^{d}(\omega):=\min \left\{l>n: \omega_{l}=d\right\}-n
$$

and

$$
R_{n}(\omega):=\max \left\{R_{n}^{d}(\omega): d \in \mathcal{D}\right\} .
$$

Lemma 2.1. Let $\mu$ be a finite Borel measure on $\Sigma$ and $\nu:=\mu \circ \Pi^{-1}$ the corresponding projection on $\Lambda$.

(i) Suppose $\mathcal{D}$ is two-dimensional. Then for all $x=\Pi(\omega) \in \Lambda$ with $\lim _{n \rightarrow \infty} \frac{R_{n}(\omega)}{n}=0$,

$$
\liminf _{r \rightarrow 0} \frac{\log \nu(B(x, r))}{\log r} \geq \liminf _{n \rightarrow \infty} \frac{\log \mu\left(B_{n}(\omega)\right)}{\log \prod_{\nu=1}^{n} b_{\nu}} .
$$

(ii) For all $x=\Pi(\omega) \in \Lambda$,

$$
\liminf _{r \rightarrow 0} \frac{\log \nu(B(x, r))}{\log r} \leq \liminf _{n \rightarrow \infty} \frac{\log \mu\left(B_{n}(\omega)\right)}{\log \prod_{\nu=1}^{n} b_{\nu}} .
$$

Proof. By Lipchitz equivalence it suffices to prove the lemma with respect to the maximum norm on $\mathbb{R}^{2}$.

To prove (i) we first suppose that $\mathcal{D}$ is two-dimensional and fix $x=\Pi(\omega) \in \Lambda$ with $\lim _{n \rightarrow \infty} \frac{R_{n}(\omega)}{n}=0$. Now the horizontal projection $\chi^{h}(x)$ is contained within $f_{\omega \mid L_{n}(\omega)+R_{L_{n}(\omega)}(\omega)}([0,1])$. Since $\mathcal{D}$ is two dimensional, there exists $d_{1}=\left(i_{1}, j_{1}\right) \in \mathcal{D}$ and $d_{2}=\left(i_{2}, j_{2}\right) \in \mathcal{D}$ such that $i_{1}=i_{1}$ and $j_{1} \neq j_{2}$ and without loss of generality we may suppose that $f_{d_{1}}(1) \leq f_{d_{2}}(0)$. By the definition of $R_{n}(\omega)$ both $d_{1}$ and $d_{2}$ occur within the finite string $\eta_{0}:=$ $\omega_{L_{n}(\omega)+1} \cdots \omega_{L_{n}(\omega)+R_{L_{n}(\omega)}}$. Now let $\eta_{1}$ be the string $\eta_{0}$ but with an extra occurrence of $d_{1}$ in place of the first occurrence of $d_{2}$ and similarly let $\eta_{2}$ be 
$\eta_{0}$ but with an extra occurrence of $d_{2}$ in place of the first occurrence of $d_{1}$. Now consider the three intervals

$$
f_{\omega \mid L_{n}(\omega)} \circ f_{\eta_{1}}([0,1]) \quad f_{\omega \mid L_{n}(\omega)} \circ f_{\eta_{0}}([0,1]) \quad f_{\omega \mid L_{n}(\omega)} \circ f_{\eta_{2}}([0,1]) .
$$

Each interval is of width at least $\prod_{\nu=1}^{L_{n}(\omega)} a_{i_{\nu} j_{\nu}} \times a_{\min }^{R_{L_{n}(\omega)}(\omega)} \geq \prod_{\nu=1}^{n} b_{i_{\nu}} \times$ $a_{\min }^{R_{L_{n}(\omega)}(\omega)+1}$ and is contained within the interval $f_{\omega \mid L_{n}(\omega)}([0,1])$. Since the three intervals have disjoint interior and $\chi^{h}(x)$ is contained within the middle one, it follows that $\chi^{h}(x)$ is at least $\prod_{\nu=1}^{n} b_{i_{\nu}} \times a_{\min }^{R_{L_{n}(\omega)}(\omega)+1}$ away from both the left and the right end points of $f_{\omega \mid L_{n}(\omega)}([0,1])$. Similarly using the existence of $\left(i_{3}, j_{3}\right),\left(i_{4}, j_{4}\right) \in \mathcal{D}$ with $i_{3} \neq i_{4}$ we may show that $\chi^{v}(x)$ is at least $\prod_{\nu=1}^{n} b_{i_{\nu}} \times a_{\min }^{R_{n}(\omega)}$ away from both the left and the right end points of $g_{\mathbf{i} \mid n}([0,1])$. Thus, we have

$$
\Pi\left(B\left(x, \prod_{\nu=1}^{n} b_{i_{\nu}} \times a_{\min }^{\max \left\{R_{L_{n}(\omega)}(\omega)+1, R_{n}(\omega)\right\}}\right)\right) \subseteq \Delta_{n}(\omega) .
$$

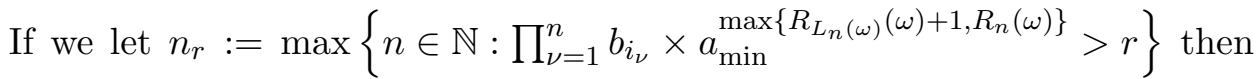
$\Pi^{-1}(B(x, r)) \subseteq B_{n_{r}}(\omega)$ whilst $\prod_{\nu=1}^{n_{r}} b_{i_{\nu}} \times a_{\min }^{\max \left\{R_{L_{n_{r}+1}(\omega)}(\omega), R_{n_{r}+1}(\omega)\right\}+2} \leq r$. Hence,

$\frac{\log \nu(B(x, r))}{\log r} \geq \frac{\log \mu\left(B_{n_{r}}(\omega)\right)}{\log \prod_{\nu=1}^{n_{r}} b_{i_{\nu}}+\max \left\{R_{L_{n_{r}+1}(\omega)}(\omega), R_{n_{r}+1}(\omega)\right\}+2 \log a_{\min }}$.

Since $\lim _{n \rightarrow \infty} \frac{R_{n}(\omega)}{n}=0$ and $\liminf _{n \rightarrow \infty} \frac{L_{n}(\omega)}{n}>0$, (i) follows.

For (ii) we begin by fixing $x=\Pi(\omega) \in \Lambda$. For each $n \in \mathbb{N}$ the image $\Pi\left(B_{n}(\omega)\right)$ contains $x$ and has diameter not exceeding $\prod_{\nu=1}^{n} b_{i_{\nu}}$. Thus $\Pi\left(B_{n}(\omega)\right) \subseteq B\left(x ; \prod_{\nu=1}^{n} b_{i_{\nu}}\right)$ and hence

$$
\frac{\log \nu\left(B\left(x ; \prod_{\nu=1}^{n} b_{i_{\nu}}\right)\right)}{\log \prod_{\nu=1}^{n} b_{i_{\nu}}} \leq \frac{\log \mu\left(B_{n}(\omega)\right)}{\log \prod_{\nu=1}^{n} b_{i_{\nu}}} .
$$

Letting $n \rightarrow \infty$ proves the lemma.

Recall the following results from geometric measure theory.

Lemma 2.2. Let $\nu$ be a finite Borel measure on some metric space $X$.

(1) Suppose we have $J \subseteq X$ with $\nu(J)>0$ such that for all $x \in J$

$$
\liminf _{r \rightarrow 0} \frac{\log \nu(B(x, r))}{\log r} \geq d
$$

Then $\operatorname{dim}_{\mathcal{H}} J \geq d$.

(2) Suppose we have $J \subseteq X$ such that for all $x \in J$

$$
\liminf _{r \rightarrow 0} \frac{\log \nu(B(x, r))}{\log r} \leq d .
$$

Then $\operatorname{dim}_{\mathcal{H}} J \leq d$. 
Proof. See [6] Proposition 2.2.

Lemma 2.3. Let $\mu$ be a finite Borel measure on $\Sigma$.

(1) Suppose $\mathcal{D}$ is two dimensional and we have $S \subseteq \Sigma$ with $\mu(S)>0$ such that for all $\omega \in S$

$$
\lim _{n \rightarrow \infty} \frac{R_{n}(\omega)}{n}=0 \quad \text { and } \quad \liminf _{n \rightarrow \infty} \frac{\log \mu\left(B_{n}(\omega)\right)}{\log \prod_{\nu=1}^{n} b_{\nu}} \geq d .
$$

Then $\operatorname{dim}_{\mathcal{H}} \Pi(S) \geq d$.

(2) Suppose we have $S \subseteq \Sigma$ such that for all $\omega \in S$

$$
\liminf _{n \rightarrow \infty} \frac{\log \mu\left(B_{n}(\omega)\right)}{\log \prod_{\nu=1}^{n} b_{\nu}} \leq d
$$

Then $\operatorname{dim}_{\mathcal{H}} \Pi(S) \leq d$.

Proof. Combine Lemma 2.1 with Lemma 2.2 .

Lemma 2.3 (i) will be used for the lower bound and Lemma 2.3 (ii) for the upper bound.

\section{Proof OF THE LOWER BOUND}

The desired lower bound is a supremum of $D_{L Y}(\mu)$ over certain invariant measures. In order to obtain a dimension estimate we need to apply Birkhoff's ergodic theorem, so we must approximate invariant measures by ergodic ones. However, these approximations have an error term which may cause them to be supported by the wrong level set. In order to obtain the correct lower bound we follow the approach of Gelfert and Rams in [9] and construct a measure which behaves asymptotically like increasingly accurate ergodic approximations to a given invariant measure.

Throughout the proof of the lower bound we fix some $\alpha \in\left[\alpha_{\min }(\varphi), \alpha_{\max }(\varphi)\right]$ and some $\mu \in \mathcal{M}_{\sigma}(\Sigma)$ satisfying $\int \varphi d \mu=\alpha$. We shall show that $\operatorname{dim}_{\mathcal{H}} \Lambda_{\alpha}^{\varphi} \geq$ $D_{L Y}(\mu)$.

For each $k \in \mathbb{N}$ we let $\tilde{\mu}_{k} \in B_{\sigma^{k}}(\Sigma)$ denote the $k$-th level approximation of $\mu$. That is, given a cylinder $\left[\omega_{1} \cdots \omega_{n k}\right]$ of length $n k$ we let

$$
\tilde{\mu}_{k}\left(\left[\omega_{1} \cdots \omega_{n k}\right]\right):=\prod_{l=0}^{n-1} \mu\left(\left[\omega_{l k+1} \cdots \omega_{l k+k}\right]\right) .
$$

Lemma 3.1. There exists a sequence $\left\{\mu_{k}\right\}$ of measures $\mu_{k} \in B_{\sigma^{k}}(\Sigma)$ satisfying,

(i) $\lim _{k \rightarrow \infty} \frac{1}{k} h\left(\mu_{k}, \sigma^{k}\right)=h(\mu, \sigma)$

(ii) $\lim _{k \rightarrow \infty} \frac{1}{k} h^{v}\left(\mu_{k}, \sigma^{k}\right)=h^{v}(\mu, \sigma)$

(iii) $\lim _{k \rightarrow \infty} \frac{1}{k} \lambda\left(\mu_{k}, \sigma^{k}\right)=\lambda(\mu, \sigma)$ 
(iv) $\lim _{k \rightarrow \infty} \frac{1}{k} \lambda^{v}\left(\mu_{k}, \sigma^{k}\right)=\lambda^{v}(\mu, \sigma)$

(v) $\lim _{k \rightarrow \infty} \int A_{k} \varphi d \mu_{k}=\alpha$.

(vi) For each $k \in \mathbb{N}$ and $\left(\omega_{1}, \cdots, \omega_{k}\right) \in \mathcal{D}^{k}$ we have $\mu_{k}\left(\left[\omega_{1} \cdots \omega_{k}\right]\right)>0$.

Proof. We begin by observing that parts $(i)-(v)$ are satisfied by $\left\{\tilde{\mu}_{k}\right\}$, the sequence of $k$ th level approximations to $\mu$. Indeed parts $(i)$ and $(i i)$ follow from the Kolmogorov-Sinai theorem (see [19] Theorem 4.18). Since $\mu$ is $\sigma$ invariant with $\mu$ and $\tilde{\mu_{k}}$ agreeing on cylinders of length $k$ we have $\lambda(\mu, \sigma)=$ $1 / k \lambda\left(\mu, \sigma^{k}\right)=1 / k \lambda\left(\tilde{\mu}_{k}, \sigma^{k}\right)$. (iv) may be proved similarly. To see $(v)$ we note that by $\sigma$ invariance of $\mu, \int A_{k}(\varphi) d \mu=\alpha$ and since $\mu$ and $\tilde{\mu}_{k}$ agree on cylinders of length $k$ we have $\left|\int A_{k}(\varphi) d \tilde{\mu}_{k}-\int A_{k}(\varphi) d \mu\right| \leq \operatorname{var}_{k} A_{k}(\varphi)$. Moreover, by the continuity of $\varphi \operatorname{var}_{k} A_{k}(\varphi) \rightarrow 0$ as $k \rightarrow \infty$.

To obtain $\left\{\mu_{k}\right\}$ with $\mu_{k} \in B_{\sigma^{k}}(\Sigma)$ satisfying (vi) in addition to conditions $(i)-(v)$ we perturb each $\tilde{\mu}_{k}$ by a small amount to obtain $\mu_{k}$ with $\mu_{k}\left(\left[\omega_{1} \cdots \omega_{k}\right]\right)>0$ for each $\left(\omega_{1}, \cdots, \omega_{k}\right) \in \mathcal{D}^{k}$ whilst using continuity to insure that

$$
\begin{aligned}
\left|h\left(\mu_{k}, \sigma^{k}\right)-h\left(\tilde{\mu}_{k}, \sigma^{k}\right)\right| & <\frac{1}{k} \\
\left|h^{v}\left(\mu_{k}, \sigma^{k}\right)-h^{v}\left(\tilde{\mu}_{k}, \sigma^{k}\right)\right| & <\frac{1}{k} \\
\left|\lambda\left(\mu_{k}, \sigma^{k}\right)-\lambda\left(\tilde{\mu}_{k}, \sigma^{k}\right)\right| & <\frac{1}{k} \\
\left|\lambda^{v}\left(\mu_{k}, \sigma^{k}\right)-\lambda^{v}\left(\tilde{\mu}_{k}, \sigma^{k}\right)\right| & <\frac{1}{k} \\
\left|\int A_{k} \varphi d \mu_{k}-\int A_{k} \varphi d \tilde{\mu}_{k}\right| & <\frac{1}{k} .
\end{aligned}
$$

Now choose $\delta_{q}>0$ for each $q \in \mathbb{N}$ in such a way that $\prod_{q=1}^{\infty}\left(1-\delta_{q}\right)>0$.

Lemma 3.2. For each $q \in \mathbb{N}$ we may choose $k(q), B(q), N(q) \in \mathbb{N}$ and $S_{q} \subseteq \Sigma$ with $\mu_{k(q)}\left(S_{q}\right)>1-\delta_{q}$ such that for all $\omega=\left(i_{\nu}, j_{\nu}\right)_{\nu \in \mathbb{N}} \in S_{q}$ and $n \in \mathbb{N}$ we have

$$
\begin{aligned}
& \text { (i) } \frac{1}{n k(q)} \log \mu_{k(q)}\left(\left[\omega_{1} \cdots \omega_{n k(q)}\right]\right)>-B(q) \\
& \text { (ii) } \frac{1}{n k(q)} \log \mu_{k(q)}\left(\left[i_{1} \cdots i_{n k(q)}\right]\right)>-B(q) \\
& \text { (iii) } \frac{R_{n}(\omega)}{n}<B(q)
\end{aligned}
$$

and for all $n \geq N(q)$ we have 


$$
\begin{aligned}
& \text { (iv) }\left|\frac{1}{n k(q)} \log \mu_{k(q)}\left(\left[\omega_{1} \cdots \omega_{n k(q)}\right]\right)+h(\mu, \sigma)\right|<\frac{1}{q} \\
& \text { (v) }\left|\frac{1}{n k(q)} \log \mu_{k(q)}\left(\left[i_{1} \cdots i_{n k(q)}\right]\right)+h^{v}(\mu, \sigma)\right|<\frac{1}{q} \\
& \text { (vi) }\left|\frac{1}{n k(q)} \log \prod_{\nu=1}^{n k(q)} a_{i_{\nu} j_{\nu}}+\lambda(\mu, \sigma)\right|<\frac{1}{q} \\
& \text { (vii) }\left|\frac{1}{n k(q)} \log \prod_{\nu=1}^{n k(q)} b_{i_{\nu}}+\lambda^{v}(\mu, \sigma)\right|<\frac{1}{q} \\
& \text { (viii) }\left|\frac{1}{n k(q)} \sum_{l=0}^{n k(q)-1} \varphi\left(\sigma^{l} \omega\right)-\alpha\right|<\frac{1}{q} \\
& \text { (ix) } \frac{R_{n}(\omega)}{n}<\frac{1}{q} \text {. }
\end{aligned}
$$

Proof. Fix $q \in \mathbb{N}$. By Lemma 3.1 (i)-(v) we may choose $k(q) \in \mathbb{N}$ so that

$$
\begin{aligned}
\left|\frac{1}{k(q)} h\left(\mu_{k(q)}, \sigma^{k(q)}\right)-h(\mu, \sigma)\right| & <\frac{1}{2 q} \\
\left|\frac{1}{k(q)} h^{v}\left(\mu_{k(q)}, \sigma^{k(q)}\right)-h^{v}(\mu, \sigma)\right| & <\frac{1}{2 q} \\
\left|\int A_{k(q)} \varphi d \mu_{k(q)}-\alpha\right| & <\frac{1}{2 q} \\
\left|\frac{1}{k(q)} \lambda\left(\mu_{k(q)}, \sigma^{k(q)}\right)-\lambda(\mu, \sigma)\right| & <\frac{1}{2 q} \\
\left|\frac{1}{k(q)} \lambda^{v}\left(\mu_{k(q)}, \sigma^{k(q)}\right)-\lambda^{v}(\mu, \sigma)\right| & <\frac{1}{2 q}
\end{aligned}
$$

Noting that $\mu_{k(q)} \in \mathcal{B}_{\sigma^{k(q)}}(\Sigma)$ is ergodic with respect to $\sigma^{k(q)}$ we may apply Birkhoff's ergodic theorem to obtain $\mu_{k(q)}$ almost everywhere convergences

$$
\begin{aligned}
\lim _{n \rightarrow \infty} \frac{1}{n} \log \mu_{k(q)}\left(\left[\omega_{1} \cdots \omega_{n k(q)}\right]\right) & =-h\left(\mu_{k(q)}, \sigma^{k(q)}\right) \\
\lim _{n \rightarrow \infty} \frac{1}{n} \log \mu_{k(q)}\left(\left[i_{1} \cdots i_{n k(q)}\right]\right) & =-h^{v}\left(\mu_{k(q)}, \sigma^{k(q)}\right) \\
\lim _{n \rightarrow \infty} \frac{1}{n} \log \prod_{\nu=1}^{n k(q)} a_{i_{\nu} j_{\nu}} & =-\lambda\left(\mu_{k(q)}, \sigma^{k(q)}\right) \\
\lim _{n \rightarrow \infty} \frac{1}{n} \log \prod_{\nu=1}^{n k(q)} b_{i_{\nu}} & =-\lambda^{v}\left(\mu_{k(q)}, \sigma^{k(q)}\right) \\
\lim _{n \rightarrow \infty} \frac{1}{n} \sum_{l=0}^{n k(q)-1} \varphi\left(\sigma^{l} \omega\right) & =\int A_{k(q)} \varphi d \mu_{k(q)} .
\end{aligned}
$$


and for each $\left(\tau_{1}, \cdots, \tau_{k(q)}\right) \in \mathcal{D}^{k(q)}$ and $\mu_{k(q)}$ almost every $\omega \in \Sigma$

$$
\begin{gathered}
\lim _{n \rightarrow \infty} \frac{\#\left\{l \in\{0, \cdots, n-1\}: \omega_{l k(q)+1} \cdots \omega_{l k(q)+k(q)}=\tau_{1} \cdots \tau_{k(q)}\right\}}{n} \\
=\mu_{k(q)}\left(\left[\tau_{1} \cdots \tau_{k(q)}\right]\right)>0 .
\end{gathered}
$$

For each of the limits (3.17) to exist we must have

$$
\lim _{n \rightarrow \infty} \frac{R_{n k(q)}^{d}(\omega)}{n k(q)}=0 .
$$

Noting the definition of $R_{n}(\omega)$ along with the fact that $R_{n k(q)-l}(\omega) \leq$ $R_{n k(q)}-l$ for $0 \leq l \leq k(q)$ we have

$$
\lim _{n \rightarrow \infty} \frac{R_{n}(\omega)}{n}=0
$$

for $\mu_{k(q)}$ almost every $\omega \in \Sigma$.

By Egorov's theorem, we may take a set $S_{q} \subseteq \Sigma$ with $\mu_{k(q)}\left(S_{q}\right)>1-\delta_{q}$ upon which each of the convergences (3.12), (3.13), (3.14), (3.15), (3.16) and (3.20) is uniform. In particular, by taking $B(q) \in \mathbb{N}$ sufficiently large we have

$$
\begin{aligned}
\frac{1}{n k(q)} \log \mu_{k(q)}\left(\left[\omega_{1} \cdots \omega_{n k(q)}\right]\right) & >-B(q) \\
\frac{1}{n k(q)} \log \mu_{k(q)}\left(\left[i_{1} \cdots i_{n k(q)}\right]\right) & >-B(q) \\
\frac{R_{n}(\omega)}{n} & <B(q)
\end{aligned}
$$

for all $n \in \mathbb{N}$ and all $\omega \in S_{q}$. Moreover by taking $N(q) \in \mathbb{N}$ sufficiently large we have

$$
\begin{aligned}
\left|\frac{1}{n k(q)} \log \mu_{k(q)}\left(\left[\omega_{1} \cdots \omega_{n k(q)}\right]\right)+\frac{1}{k(q)} h\left(\mu_{k(q)}, \sigma^{k(q)}\right)\right| & <\frac{1}{2 q} \\
\left|\frac{1}{n k(q)} \log \mu_{k(q)}\left(\left[i_{1} \cdots i_{n k(q)}\right]\right)+\frac{1}{k(q)} h^{v}\left(\mu_{k(q)}, \sigma^{k(q)}\right)\right| & <\frac{1}{2 q} \\
\left|\frac{1}{n k(q)} \log \prod_{\nu=1}^{n k(q)} a_{i_{\nu} j_{\nu}}+\frac{1}{k(q)} \lambda\left(\mu_{k(q)}, \sigma^{k(q)}\right)\right| & <\frac{1}{2 q} \\
\left|\frac{1}{n k(q)} \log \prod_{\nu=1}^{n k(q)} b_{i_{\nu}}+\frac{1}{k(q)} \lambda^{v}\left(\mu_{k(q)}, \sigma^{k(q)}\right)\right| & <\frac{1}{2 q} \\
\left|\frac{1}{n k(q)} \sum_{l=0}^{n k(q)-1} \varphi\left(\sigma^{l} \omega\right)-\int A_{k(q)} \varphi d \mu_{k(q)}\right| & <\frac{1}{2 q} \\
\frac{R_{n}(\omega)}{n} & <\frac{1}{q}
\end{aligned}
$$


for all $n \geq N(q)$ and all $\omega \in S_{q}$. Combining these inequalities with the inequalities in (3.7), (3.8) and (3.9) proves the lemma.

We now construct our measure $\mathcal{W}$. First define a rapidly increasing sequence $\left(\gamma_{q}\right)_{q \in \mathbb{N} \cup\{0\}}$ of natural numbers by $\gamma_{0}=0, \gamma_{1}=1$ and for $q>1$ we let

$$
\gamma_{q}:=q \gamma_{q-1}\left(\prod_{l=1}^{q+1} N(l)\right)\left(\prod_{l=1}^{q+1} B(l)\right)\left(\prod_{l=1}^{q+1} k(l)\right)+\gamma_{q-1} .
$$

We now define a measure $\mathcal{W}$ on $\Sigma$ by first defining $\mathcal{W}$ on a semi-algebra of cylinders and then extending $\mathcal{W}$ to a Borel probability measure on $\Sigma$ via the Daniell-Kolmogorov consistency theorem ([19] Theorem 0.5). Given a cylinder $\left[\omega_{1} \cdots \omega_{\gamma_{Q}}\right]$ of length $\gamma_{Q}$ for some $Q \in \mathbb{N}$ we define

$$
\mathcal{W}\left(\left[\omega_{1} \cdots \omega_{\gamma_{Q}}\right]\right):=\prod_{q=1}^{Q} \mu_{k(q)}\left(\left[\omega_{\gamma_{q-1}+1} \cdots \omega_{\gamma_{q}}\right]\right) .
$$

Define $S \subseteq \Sigma$ by

$$
S:=\bigcap_{q=1}^{\infty}\left\{\omega \in \Sigma:\left[\omega_{\gamma_{q-1}+1} \cdots \omega_{\gamma_{q}}\right] \cap S_{q} \neq \emptyset\right\} .
$$

Lemma 3.3. $\mathcal{W}(S)>0$.

Proof. $\mathcal{W}(S) \geq \prod_{q=1}^{\infty} \mu_{q}\left(S_{q}\right)>\prod_{q=1}^{\infty}\left(1-\delta_{q}\right)>0$.

Lemma 3.4. For all $\omega \in S$ we have

(i) $\lim _{n \rightarrow \infty} \frac{1}{n} \log \prod_{\nu=1}^{n} a_{i_{\nu} j_{\nu}}=-\lambda(\mu, \sigma)$

(ii) $\lim _{n \rightarrow \infty} \frac{1}{n} \log \prod_{\nu=1}^{n} b_{i_{\nu}}=-\lambda^{v}(\mu, \sigma)$

(iii) $\lim _{n \rightarrow \infty} \frac{1}{n} \sum_{l=0}^{n-1} \varphi\left(\sigma^{l} \omega\right)=\alpha$.

Proof. We shall prove part (iii). The proofs for parts (i) and (ii) are similar. Fix $\omega \in S$ and choose for each $q \in \mathbb{N}$ some $\tau^{q} \in \Sigma$ such that $\sigma^{\gamma_{q-1}} \tau_{q} \in$ $\left[\omega_{\gamma_{q-1}+1} \cdots \omega_{\gamma_{q}}\right] \cap S_{q}$. Given $n \in \mathbb{N}$ we choose $q_{n}$ so that $\gamma_{q_{n}} \leq n$ is maximal. Since $\gamma_{q_{n}}-\gamma_{q_{n}-1} \geq N\left(q_{n}\right)$ and $\gamma_{q_{n}}-\gamma_{q_{n}-1} \leq n$ we have

$$
\left|\sum_{l=\gamma_{q_{n}}-1}^{\gamma_{q_{n}}-1} \varphi\left(\sigma^{l} \tau^{q_{n}}\right)-\left(\gamma_{q_{n}}-\gamma_{q_{n}-1}\right) \alpha\right|<\frac{n}{q_{n}}
$$

by Lemma 3.2 (viii). So by our choice of $\tau^{q}$ and $\gamma_{q_{n}}-\gamma_{q_{n}-1} \leq n$ we have

$$
\left|\sum_{l=\gamma_{q_{n}}-1}^{\gamma_{q_{n}}-1} \varphi\left(\sigma^{l} \omega\right)-\left(\gamma_{q_{n}}-\gamma_{q_{n}-1}\right) \alpha\right|<\frac{n}{q_{n}}+\sum_{l=0}^{n-1} \operatorname{var}_{l}(\varphi) .
$$


By the construction of $\left(\gamma_{q}\right)_{q \in \mathbb{N}}, \gamma_{q_{n}-1} \leq \gamma_{q_{n}} / q_{n} \leq n / q_{n}$ and hence

$$
\left|\sum_{l=0}^{\gamma_{q_{n}-1}-1} \varphi\left(\sigma^{l} \omega\right)-\gamma_{q_{n}-1} \alpha\right|<\frac{n}{q_{n}}\left(\|\varphi\|_{\infty}+\alpha\right) .
$$

Now either $n-\gamma_{q_{n}} \geq N\left(q_{n}+1\right)$ or $n-\gamma_{q_{n}} \leq N\left(q_{n}+1\right)$. In the former case we reason as in (3.32) and (3.33) to obtain

$$
\left|\sum_{l=\gamma_{q_{n}}}^{n-1} \varphi\left(\sigma^{l} \omega\right)-\left(n-\gamma_{q_{n}}\right) \alpha\right|<\frac{n}{q_{n}}+\sum_{l=0}^{n-1} \operatorname{var}_{l}(\varphi) .
$$

In the latter case, by the construction of $\left(\gamma_{q}\right)_{q \in \mathbb{N}}$ we have $N\left(q_{n}+1\right) \leq$ $\gamma_{q_{n}} / q_{n} \leq n / q_{n}$ and hence

$$
\left|\sum_{l=\gamma_{q_{n}}}^{n-1} \varphi\left(\sigma^{l} \omega\right)-\left(n-\gamma_{q_{n}}\right) \alpha\right|<\frac{n}{q_{n}}\left(\|\varphi\|_{\infty}+\alpha\right) .
$$

Thus, by (3.33), 3.34), 3.35), 3.36) we have

$$
\left|\sum_{l=0}^{n-1} \varphi\left(\sigma^{l} \omega\right)-\alpha\right| \leq \frac{2}{q_{n}}+\frac{2}{n} \sum_{l=0}^{n} \operatorname{var}_{l} \varphi+\left(\|g\|_{\infty}+|\alpha|\right) \frac{2}{q_{n}}
$$

Note that since $\varphi$ is continuous we have $\frac{1}{n} \sum_{l=0}^{n} \operatorname{var}_{l}(\varphi) \rightarrow 0$. Thus, dividing by $n$ and letting $n \rightarrow \infty$ proves the lemma.

Lemma 3.5. $S \subseteq \Sigma_{\alpha}^{\varphi}$.

Proof. $S \subseteq \Sigma_{\alpha}$ is precisely Lemma 3.4 (iii).

Lemma 3.6. For all $\omega=\left(i_{\nu}, j_{\nu}\right)_{\nu \in \mathbb{N}} \in S$ we have

(i) $\lim _{n \rightarrow \infty} \frac{1}{n} \log \mathcal{W}\left(\left[\omega_{1} \cdots \omega_{n}\right]\right)=-h(\mu, \sigma)$

(ii) $\lim _{n \rightarrow \infty} \frac{1}{n} \log \mathcal{W}\left(\left[i_{1} \cdots i_{n}\right]\right)=-h^{v}(\mu, \sigma)$.

Proof. Both proofs resemble that of Lemma 3.4. We prove only part $(i)$ since the proof of part $(i i)$ is similar.

Take $\omega \in S$. Given $n \in \mathbb{N}$ we choose $q_{n}$ so that $\gamma_{q_{n}} \leq n$ is maximal. Since $\gamma_{q_{n}}-\gamma_{q_{n}-1} \geq N\left(q_{n}\right)$ and $\gamma_{q_{n}}-\gamma_{q_{n}-1} \leq n$ we have

$$
\left|\log \mu_{k\left(q_{n}\right)}\left(\left[\omega_{\gamma_{q_{n}-1}+1} \cdots \omega_{\gamma_{q_{n}}}\right]\right)+\left(\gamma_{q_{n}}-\gamma_{q_{n}-1}\right) h(\mu, \sigma)\right|<\frac{n}{q_{n}}
$$

by Lemma 3.2 (iv). Moreover, by the construction of $\left(\gamma_{q}\right)_{q \in \mathbb{N}}$,

$$
\max \left\{B(l): l \leq q_{n}\right\} \gamma_{q_{n}-1} \leq \gamma_{q_{n}} / q_{n} \leq n / q_{n}
$$

so by Lemma 3.2 (i)

$$
\left|\sum_{l=1}^{q_{n}-1} \log \mu_{k(l)}\left(\left[\omega_{\gamma_{l-1}+1} \cdots \omega_{\gamma_{l}}\right]\right)+\gamma_{q_{n}-1} h(\mu, \sigma)\right|<\frac{n}{q} .
$$


Now either $n-\gamma_{q_{n}} \geq N\left(q_{n}+1\right)$ or $n-\gamma_{q_{n}} \leq N\left(q_{n}+1\right)$. In the former case we apply Lemma 3.2 (iv) and note that $n-\gamma_{q_{n}} \leq n$ to obtain

$$
\left|\log \mu_{k\left(q_{n}+1\right)}\left(\left[\omega_{\gamma_{q_{n}}} \cdots \omega_{n}\right]\right)+\left(n-\gamma_{q_{n}}\right) h(\mu, \sigma)\right|<\frac{n}{q_{n}+1} .
$$

In the latter case, by the construction of $\left(\gamma_{q}\right)_{q \in \mathbb{N}}$ we have $N\left(q_{n}+1\right) \leq$ $\gamma_{q_{n}} / q_{n} \leq n / q_{n}$ and hence

$$
\left|\log \mu_{k\left(q_{n}+1\right)}\left(\left[\omega_{\gamma_{q_{n}}} \cdots \omega_{n}\right]\right)+\left(n-\gamma_{q_{n}}\right) h(\mu, \sigma)\right|<\frac{n}{q_{n}} .
$$

Thus, by (3.38), (3.40), 3.41), 3.42 together with the construction of $\mathcal{W}$ we have

$$
\left|\log \mathcal{W}\left(\left[\omega_{1} \cdots \omega_{n}\right]\right)+n h(\mu, \sigma)\right|<\frac{3 n}{q_{n}} .
$$

Dividing by $n$ and letting $n \rightarrow \infty$ proves the lemma.

Lemma 3.7. For all $\omega \in S$ we have $\lim _{n \rightarrow \infty} \frac{R_{n}(\omega)}{n}=0$.

Proof. This follows from Lemma 3.2 (iii) and (ix) in a similar way to the proof of Lemma 3.6 .

Lemma 3.8. For all $\omega \in S$

$$
\liminf _{n \rightarrow \infty} \frac{\log \mathcal{W}\left(B_{n}(\omega)\right)}{\log \prod_{\nu=1}^{n} b_{i_{\nu}}} \geq D_{L Y}(\mu) .
$$

Proof. Fix $\omega=\left(i_{\nu}, j_{\nu}\right)_{\nu \in \mathbb{N}} \in S$. By $(2.2)$ we have

$$
\lim _{n \rightarrow \infty} \frac{1}{n} \log \prod_{\nu=1}^{L_{n}(\omega)} a_{\omega_{\nu}}=\lim _{n \rightarrow \infty} \frac{1}{n} \log \prod_{\nu=1}^{n} b_{i_{\nu}} .
$$

Hence, by Lemma 3.4 (i) and (ii) we have

$$
\lim _{n \rightarrow \infty} \frac{L_{n}(\omega)}{n}=\frac{\lambda^{v}(\mu, \sigma)}{\lambda(\mu, \sigma)}
$$

Given $n \in \mathbb{N}$ let $q_{n}$ be the greatest integer satisfying $\gamma_{q_{n}-1}<L_{n}(\omega)$ and let $L_{n}^{+}(\omega):=\min \left\{l \geq L_{n}(\omega): k\left(q_{n}\right) \mid\left(l-\gamma_{q_{n}-1}\right)\right\}$. By the construction of $\left(\gamma_{q}\right)_{q \in \mathbb{N}}$ we have $k\left(q_{n}\right) \leq \gamma_{q_{n}-1} / q_{n} \leq L_{n}(\omega) / q_{n} \leq n / q_{n}$ and so by 3.45

$$
\lim _{n \rightarrow \infty} \frac{L_{n}^{+}(\omega)}{n}=\lim _{n \rightarrow \infty} \frac{L_{n}(\omega)+o\left(k\left(q_{n}\right)\right)}{n}=\lim _{n \rightarrow \infty} \frac{L_{n}(\omega)}{n}=\frac{\lambda^{v}(\mu, \sigma)}{\lambda(\mu, \sigma)} .
$$

Moreover, $L_{n}^{+}(\omega) \geq L_{n}(\omega)$ so

$$
B_{n}(\omega) \subseteq\left[\omega_{1} \cdots \omega_{L_{n}^{+}(\omega)}\right] \cap \sigma^{-L_{n}^{+}(\omega)}\left[i_{L_{n}^{+}(\omega)} \cdots i_{n}\right] .
$$

Thus, by Lemma 3.4 (ii) it suffices to show that

$$
\lim _{n \rightarrow \infty} \frac{1}{n} \mathcal{W}\left(\left[\omega_{1} \cdots \omega_{L_{n}^{+}(\omega)}\right] \cap \sigma^{-L_{n}^{+}(\omega)}\left[i_{L_{n}^{+}(\omega)} \cdots i_{n}\right]\right)=\frac{\lambda^{v}(\mu, \sigma)}{\lambda(\mu, \sigma)} h(\mu, \sigma)+\left(1-\frac{\lambda^{v}(\mu, \sigma)}{\lambda(\mu, \sigma)}\right) h^{v}(\mu, \sigma) .
$$


Now since $L_{n}^{+}(\omega)-\gamma_{q_{n}-1}$ is a multiple of $k\left(q_{n}\right)$ it follows from the construction of $\mathcal{W}$ that for all $\tau=\left(\tau_{\nu}\right)_{\nu=1}^{n} \in \mathcal{D}^{n}$ we have

$$
\mathcal{W}\left(\left[\tau_{1} \cdots \tau_{n}\right]\right)=\mathcal{W}\left(\left[\tau_{1} \cdots \tau_{L_{n}^{+}(\omega)}\right]\right) \mathcal{W}\left(\left[\tau_{L_{n}^{+}(\omega)+1} \cdots \tau_{n}\right]\right) .
$$

Hence, it suffices to show that

$$
\begin{aligned}
\lim _{n \rightarrow \infty} \frac{1}{n} \mathcal{W}\left(\left[\omega_{1} \cdots \omega_{L_{n}^{+}(\omega)}\right]\right) & =\frac{\lambda^{v}(\mu, \sigma)}{\lambda(\mu, \sigma)} h(\mu, \sigma) \\
\lim _{n \rightarrow \infty} \frac{1}{n} \mathcal{W}\left(\sigma^{-L_{n}^{+}(\omega)}\left[i_{L_{n}^{+}(\omega)} \cdots i_{n}\right]\right) & =\left(1-\frac{\lambda^{v}(\mu, \sigma)}{\lambda(\mu, \sigma)}\right) h^{v}(\mu, \sigma) .
\end{aligned}
$$

Equation (3.50) follows from Lemma 3.6 (i) combined with 3.46). Equation (3.50) follows from Lemma 3.6 (ii) and (3.46) along with

$$
\mathcal{W}\left(\left[i_{1} \cdots i_{n}\right]\right)=\mathcal{W}\left(\left[i_{1} \cdots i_{L_{n}^{+}(\omega)}\right]\right) \mathcal{W}\left(\left[i_{L_{n}^{+}(\omega)+1} \cdots i_{n}\right]\right)
$$

which follows from 3.49 .

Lemma 3.9. $\operatorname{dim}_{\mathcal{H}}\left(\Pi\left(\Sigma_{\alpha}^{\varphi}\right)\right) \geq D_{L Y}(\mu)$.

Proof. We begin with the special cases in which $\mathcal{D}$ is not two dimensional. If $\mathcal{D}$ is not two dimensional then either there is just one $i$ for which there exists $j$ with $(i, j) \in \mathcal{D}$, or for each $i$ there is just one $j$ for which $(i, j) \in \mathcal{D}$. In the former case we have $h^{v}(\mu, \sigma)=0$. Thus it suffices to show that

$$
\operatorname{dim}_{\mathcal{H}}\left(\Pi\left(\Sigma_{\alpha}^{\varphi}\right)\right) \geq \frac{h(\mu, \sigma)}{\lambda(\mu, \sigma)} .
$$

Moreover this follows from Olsen [15] Theorem 1 applied to the projection $\chi^{h}\left(\Lambda_{\alpha}^{\varphi}\right)$ onto the horizontal axis, together with the fact that the Hausdorff dimension cannot increase under projection. Similarly when there is just one $j$ for each $i$ we have $h(\mu, \sigma)=h^{v}(\mu, \sigma)$ and so it suffices to show

$$
\operatorname{dim}_{\mathcal{H}}\left(\Pi\left(\Sigma_{\alpha}^{\varphi}\right)\right) \geq \frac{h^{v}(\mu, \sigma)}{\lambda^{v}(\mu, \sigma)}
$$

which follows from Olsen [15] Theorem 1 applied to the projection $\chi^{v}\left(\Lambda_{\alpha}^{\varphi}\right)$ onto the vertical. Henceforth we assume that $\mathcal{D}$ is two dimensional.

By Lemma 3.5 it suffices to prove that $\operatorname{dim}_{\mathcal{H}}(\Pi(S)) \geq D_{L Y}(\mu)$. Now by Lemma 3.3 we have $\mathcal{W}(S)>0$ and by Lemma 3.8 we have

$$
\liminf _{n \rightarrow \infty} \frac{\log \mathcal{W}\left(B_{n}(\omega)\right)}{\log \prod_{\nu=1}^{n} b_{i_{\nu}}} \geq D_{L Y}(\mu)
$$

for all $\omega \in S$. Moreover, by Lemma 3.7 for all $\omega \in S$

$$
\lim _{n \rightarrow \infty} \frac{R_{n}(\omega)}{n}=0
$$

Thus, by Lemma 2.3 (i), combined with the assumption that $\mathcal{D}$ is two dimensional, the lemma holds. 
Lemma 3.9 holds for all $\mu \in \mathcal{M}_{\sigma}(\Sigma)$ satisfying $\int \varphi d \mu=\alpha$. Therefore,

$$
\operatorname{dim}_{\mathcal{H}} \Lambda_{\alpha}^{\varphi} \geq \sup \left\{D_{L Y}(\mu): \mu \in \mathcal{M}_{\sigma}(\Sigma), \int \varphi d \mu=\alpha\right\} .
$$

\section{PRoOF of THE UPPER BOUND}

We begin by demonstrating that the function $f:\left[\alpha_{\min }(\varphi), \alpha_{\max }(\varphi)\right] \rightarrow \mathbb{R}$ given by

$$
f(\alpha)=\sup \left\{D_{L Y}(\mu): \mu \in \mathcal{M}_{\sigma}(\Sigma), \int \varphi d \mu=\alpha\right\}
$$

is continuous on $\left[\alpha_{\min }(\varphi), \alpha_{\max }(\varphi)\right]$.

Lemma 4.1. $f$ is continuous on $\left[\alpha_{\min }(\varphi), \alpha_{\max }(\varphi)\right]$.

Proof. Fix $\alpha_{*} \in\left[\alpha_{\min }(\varphi), \alpha_{\max }(\varphi)\right]$. First we show that $f$ is upper semicontinuous at $\alpha^{*}$. Since $\left[\alpha_{\min }(\varphi), \alpha_{\max }(\varphi)\right]=\left\{\alpha \in \mathbb{R}: \Sigma_{\alpha}^{\varphi} \neq \emptyset\right\}$ we may take a sequence $\left\{\alpha_{n}\right\}_{n \in \mathbb{N}}$ such that $\lim _{n \rightarrow \infty} \alpha_{n}=\alpha, \lim _{n \rightarrow \infty} f\left(\alpha_{n}\right)=\limsup _{\alpha \rightarrow \alpha_{*}} f(\alpha)$ and for each $n$ there is a measure $\mu_{n}$ such that $\int \varphi d \mu_{n}=\alpha_{n}$ and $D_{L Y}\left(\mu_{n}\right)>$ $f\left(\alpha_{n}\right)-1 / n$. Since $\mathcal{M}_{\sigma}(\Sigma)$ is compact we may take a weak $*$ limit $\mu_{*}$ of $\left\{\mu_{n}\right\}_{n \in \mathbb{N}}$. It follows from the upper semi-continuity of entropy (see [19] Theorem 8.2) that $\mu \mapsto D_{L Y}(\mu)$ is upper semi-continuous. Thus, $f\left(\alpha_{*}\right) \geq$ $D_{L Y}\left(\mu_{*}\right) \geq \lim \sup _{n \rightarrow \infty} D_{L Y}\left(\mu_{n}\right) \geq \lim \sup _{\alpha \rightarrow \alpha_{*}} f(\alpha)$. Hence, $f$ is uppersemicontinuous at $\alpha^{*}$ for all $\alpha_{*} \in\left[\alpha_{\min }(\varphi), \alpha_{\max }(\varphi)\right]$.

To prove that $f$ is lower semi-continuous we first show that, provided $\alpha_{*} \neq$ $\alpha_{\max }(\varphi), \liminf _{\alpha \rightarrow \infty}^{\alpha>\alpha_{*}} f(\alpha)>f\left(\alpha_{*}\right)-\epsilon$ for an arbitrary $\epsilon>0$. Choose $\mu_{\epsilon} \in \mathcal{M}_{\sigma}(\Sigma)$ with $\int \varphi d \mu_{\epsilon}=\alpha_{*}$ and $D_{L Y}\left(\mu_{\epsilon}\right)>f\left(\alpha_{*}\right)-\epsilon$. Take $\mu_{\max } \epsilon$ $\mathcal{M}_{\sigma}(\Sigma)$ with $\int \varphi d \mu_{\max }=\alpha_{\max }$. Now for each $\rho \in(0,1)$ we let $\mu_{\rho, \epsilon}:=$ $\rho \mu_{\epsilon}+(1-\rho) \mu_{\max }$. Note that $\int f d \mu_{\rho, \epsilon}=\rho \alpha_{*}+(1-\rho) \alpha_{\max }$. Moreover, it follows from the fact that the entropy map is affine (see [19] Theorem 8.1) that $\rho \mapsto D_{L Y}\left(\mu_{\rho, \epsilon}\right)$ is continuous. Hence $f\left(\alpha_{*}\right)-\epsilon<D_{L Y}\left(\mu_{*}\right)$ $\leq \liminf _{\rho \rightarrow 1} D_{L Y}\left(\mu_{\rho, \epsilon}\right) \leq \liminf _{\rho \rightarrow 1} f\left(\rho \alpha_{*}+(1-\rho) \alpha_{+}\right)=\liminf _{\alpha \rightarrow \infty}^{\alpha>\alpha_{*}} f(\alpha)$. The proof that $\liminf \operatorname{in}_{\alpha \rightarrow \infty}^{\alpha<\alpha_{*}} f(\alpha) \geq f\left(\alpha_{*}\right)$ for all $\alpha_{*} \neq \alpha_{\min }(\varphi)$ is similar. Thus, $f$ is lower semi-continuous at $\alpha^{*}$ for all $\alpha_{*} \in\left[\alpha_{\min }(\varphi), \alpha_{\max }(\varphi)\right]$.

Thus, to show that the spectrum is continuous it suffices to identify the prove that $\operatorname{dim}_{\mathcal{H}} \Lambda_{\alpha}^{\varphi}=f(\alpha)$.

Another consequence of Lemma 4.1 is that in proving the upper bound in Theorem 1, $\operatorname{dim}_{\mathcal{H}} \Lambda_{\alpha}^{\varphi} \leq f(\alpha)$, it suffices to prove

$$
\operatorname{dim}_{\mathcal{H}} \Lambda_{\alpha}^{\varphi} \leq \sup \left\{D_{L Y}(\mu): \mu \in \mathcal{M}_{\sigma}(\Sigma),\left|\int \varphi d \mu-\alpha\right| \leq \epsilon\right\}
$$

for arbitrarily small $\epsilon>0$.

The key lemma in the proof of the upper bound is Lemma 4.2, which uses an idea from Barański 3 to give an upper estimate for the dimension of the projection of a subset of the symbolic space in terms the possible limit points for frequencies of words amongst its members. From Lemma 4.2 we 
can deduce an estimate of the form 4.2 with an error term given by the variance of a potential across sets of strings with a common first digit $\operatorname{var}_{1}(\varphi)$ (see Lemma 4.3). By iterating our system some large number of times we are able to transform this estimate into estimates of the form 4.2 with an arbitrary degree of precision (see Lemma 4.5).

We introduce the following terminology for the proof of the upper bound. Define

$$
\mathbb{P}:=\left\{\left(p_{d}\right)_{d \in \mathcal{D}} \in[0,1]^{\mathcal{D}}: \sum_{d \in \mathcal{D}} p_{d}=1\right\}
$$

be the simplex of probability vectors on the digit set $\mathcal{D}$ and

$$
\mathbb{B}:=\left\{\left(p_{d}\right)_{d \in \mathcal{D}} \in \mathbb{P}: p_{d} \in \mathbb{Q} \backslash\{0\}\right\} .
$$

Note that $\mathbb{P}$ is compact and $\mathbb{B}$ is a countable dense subset. For each $\mathbf{p} \in \mathbb{P}$ we let $\mu_{\mathbf{p}}$ denote the corresponding Bernoulli measure on $\Sigma$. Given $(i, j) \in \mathcal{D}$ we define, for each $\omega \in \Sigma$ and $n \in \mathbb{N}$

$$
N_{i j}(\omega \mid n):=\#\left\{l \in\{1, \cdots, n\}: \omega_{l}=(i, j)\right\}
$$

and $P_{i j}(\omega \mid n):=N_{i j}(\omega \mid n) / n$. This implies that for each $\omega \in \Sigma$ and $n \in \mathbb{N}$ we have a probability vector $\mathbf{P}(\omega \mid n):=\left(P_{i j}(\omega \mid n)\right)_{(i, j) \in \mathcal{D}} \in \mathbb{P}$ known as the nth level frequency vector for $\omega$. We also let $N_{i}(\omega \mid n):=\sum_{j=1}^{M_{i}} N_{i j}(\omega \mid n)$, $P_{i}(\omega \mid n):=\sum_{j=1}^{M_{i}} P_{i j}(\omega \mid n)$ and $p_{i}:=\sum_{j=1}^{M_{i}} p_{i j}$ for $\left(p_{i j}\right)_{(i, j) \in \mathcal{D}} \in \mathbb{P}$.

Lemma 4.2. Suppose we have $\Omega \subseteq \Sigma$ and $A \subseteq \mathbb{P}$ such that for all $\omega \in \Omega$ every limit point of the sequence $(\mathbf{P}(\omega \mid n))_{n \in \mathbb{N}}$ of frequency vectors for $\omega$ lies within $A$. Then $\operatorname{dim}_{\mathcal{H}} \Pi(\Omega) \leq \sup \left\{D_{L Y}\left(\mu_{p}\right): p \in A\right\}$.

Proof. Recall that $D_{L Y}(\mu):=\frac{h(\mu, \sigma)}{\lambda(\mu, \sigma)}+\left(\frac{1}{\lambda^{v}(\mu, \sigma)}-\frac{1}{\lambda(\mu, \sigma)}\right) h^{v}(\mu, \sigma)$, so for Bernoulli measures $\mu_{\mathbf{p}}$ we can write

$$
\begin{aligned}
D_{L Y}\left(\mu_{\mathbf{p}}\right) & =\frac{\sum_{(i, j) \in \mathcal{D}} p_{i j} \log p_{i j}}{\sum_{(i, j) \in \mathcal{D}} p_{i j} \log a_{i j}}+\frac{\sum_{i=1}^{N} p_{i} \log p_{i}}{\sum_{i=1}^{N} p_{i} \log b_{i}}-\frac{\sum_{i=1}^{N} p_{i} \log p_{i}}{\sum_{(i, j) \in \mathcal{D}} p_{i j} \log a_{i j}} \\
& =\frac{\sum_{(i, j) \in \mathcal{D}} p_{i j} \log p_{i j} / p_{i}}{\sum_{(i, j) \in \mathcal{D}} p_{i j} \log a_{i j}}+\frac{\sum_{i=1}^{N} p_{i} \log p_{i}}{\sum_{i=1}^{N} p_{i} \log b_{i}} .
\end{aligned}
$$

Let $s:=\sup \left\{D_{L Y}\left(\mu_{p}\right): p \in A\right\}$. Fix some $\delta>0$ and $\omega \in \Omega$. For each $n \in \mathbb{N}$ we take $\rho(n)=\left(\rho_{i j}(n)\right)_{(i, j) \in \mathcal{D}} \in \mathbb{P}$ defined by

$$
\rho_{i j}(n):=\left\{\begin{array}{l}
P_{i}(\omega \mid n) \frac{P_{i j}\left(\omega \mid L_{n}(\omega)\right)}{P_{i}\left(\omega \mid L_{n}(\omega)\right)} \text { if } P_{i j}\left(\omega \mid L_{n}(\omega)\right) \neq 0 \\
0 \text { if } P_{i j}\left(\omega \mid L_{n}(\omega)\right)=0
\end{array} .\right.
$$

Since $\mathbb{P}$ is compact we may take $n_{q}$ such that:

(i) $\lim _{q \rightarrow \infty} \frac{\sum_{i=1}^{N} P_{i}\left(\omega \mid n_{q}\right) \log P_{i}\left(\omega \mid n_{q}\right)}{\sum_{i=1}^{N} P_{i}\left(\omega \mid n_{q}\right) \log b_{i}}=\liminf _{n \rightarrow \infty} \frac{\sum_{i=1}^{N} P_{i}(\omega \mid n) \log P_{i}(\omega \mid n)}{\sum_{i=1}^{N} P_{i}(\omega \mid n) \log b_{i}}$; 
(ii) $\rho:=\lim _{q \rightarrow \infty} \rho\left(n_{q}\right)$ exists;

(iii) $\mathbf{P}:=\lim _{q \rightarrow \infty} \mathbf{P}\left(\omega \mid L_{n_{q}}(\omega)\right)$ exists.

Since $\omega \in \Omega, \mathbf{P} \in A$. Letting $b_{\max }:=\max _{i} b_{i}<1$ we may take $\beta(\omega)=$ $\left(\beta^{\omega}{ }_{i j}\right)_{(i, j) \in \mathcal{D}} \in \mathbb{B}$ such that $\rho_{i j} / \beta_{i j}^{\omega}, \beta_{i j}^{\omega} / \rho_{i j}<b_{\max }^{-\delta / 2}$ for all $(i, j) \in \mathcal{D}$ with $\rho_{i j} \neq 0$. By the definition of $B_{n_{q}}(\omega)$ we have

$$
\mu_{\beta(\omega)}\left(B_{n_{q}}(\omega)\right)=\prod_{\nu=1}^{L_{n_{q}}(\omega)} \beta_{i_{\nu} j_{\nu}}^{\omega} \times \prod_{\nu=L_{n_{q}}(\omega)+1}^{n_{q}} \beta_{i_{\nu}}^{\omega}
$$

and so

$$
\begin{aligned}
\log \mu_{\beta(\omega)}\left(B_{n_{q}}(\omega)\right) & =\sum_{(i, j) \in \mathcal{D}} N_{i j}\left(\omega \mid L_{n_{q}}(\omega)\right) \log \beta_{i j}^{\omega}+\sum_{i=1}^{N}\left(N_{i}\left(\omega \mid n_{q}\right)-N_{i}\left(\omega \mid L_{n_{q}}(\omega)\right)\right) \log \beta_{i}^{\omega} \\
& =\sum_{(i, j) \in \mathcal{D}} N_{i j}\left(\omega \mid L_{n_{q}}(\omega)\right) \log \beta_{i j}^{\omega} / \beta_{i}^{\omega}+\sum_{i=1}^{N} N_{i}\left(\omega \mid n_{q}\right) \log \beta_{i}^{\omega} .
\end{aligned}
$$

By 2.2 we have

$$
\log a_{\min } \leq \sum_{i} N_{i}\left(\omega \mid n_{q}\right) \log b_{i}-\sum_{i j} N_{i j}\left(\omega \mid L_{n_{q}}(\omega)\right) \log a_{i j} \leq 0 .
$$

Hence,

$$
\begin{aligned}
\liminf _{q \rightarrow \infty} \frac{\mu_{\beta(\omega)}\left(B_{n_{q}}(\omega)\right)}{\log \prod_{\nu=1}^{n_{q}} b_{i_{\nu}}} & \leq \liminf _{q \rightarrow \infty} \frac{\sum_{i j} N_{i j}\left(\omega \mid L_{n_{q}}(\omega)\right) \log \beta_{i j}^{\omega} / \beta_{i}^{\omega}}{\sum_{i j} N_{i j}\left(\omega \mid L_{n_{q}}(\omega)\right) \log a_{i j}}+\frac{\sum_{i} N_{i}\left(\omega \mid n_{q}\right) \log \beta_{i}^{\omega}}{\sum_{i} N_{i}\left(\omega \mid n_{q}\right) \log b_{i}} \\
& \leq \liminf _{q \rightarrow \infty} \frac{\sum_{i j} P_{i j}\left(\omega \mid L_{n_{q}}(\omega)\right) \log \beta_{i j}^{\omega} / \beta_{i}^{\omega}}{\sum_{i j} P_{i j}\left(\omega \mid L_{n_{q}}(\omega)\right) \log a_{i j}}+\frac{\sum_{i} P_{i}\left(\omega \mid n_{q}\right) \log \beta_{i}^{\omega}}{\sum_{i} P_{i}\left(\omega \mid n_{q}\right) \log b_{i}} .
\end{aligned}
$$

Since $\beta(\omega) \rho_{i j} / \beta_{i j}^{\omega}, \beta_{i j}^{\omega} / \rho_{i j}<b_{\max }^{-\delta / 2}$ for all $(i, j) \in \mathcal{D}$ such that $\rho_{i j} \neq 0$ we have

$$
\begin{array}{r}
\liminf _{q \rightarrow \infty} \frac{\sum_{i j} P_{i j}\left(\omega \mid L_{n_{q}}(\omega)\right) \log \beta_{i j}^{\omega} / \beta_{i}^{\omega}}{\sum_{i j} P_{i j}\left(\omega \mid L_{n_{q}}(\omega)\right) \log a_{i j}}+\frac{\sum_{i} P_{i}\left(\omega \mid n_{q}\right) \log \beta_{i}^{\omega}}{\sum_{i} P_{i}\left(\omega \mid n_{q}\right) \log b_{i}} \\
\leq \liminf _{q \rightarrow \infty} \frac{\sum_{i j} P_{i j}\left(\omega \mid L_{n_{q}}(\omega)\right) \log \rho_{i j}\left(n_{q}\right) / \rho_{i}\left(n_{q}\right)}{\sum_{i j} P_{i j}\left(\omega \mid L_{n_{q}}(\omega)\right) \log a_{i j}}+\frac{\sum_{i} P_{i}\left(\omega \mid n_{q}\right) \log \rho_{i}\left(n_{q}\right)}{\sum_{i} P_{i}\left(\omega \mid n_{q}\right) \log b_{i}}+\delta .
\end{array}
$$

By the definition of $\rho\left(n_{q}\right) 4.6$

$$
\begin{array}{r}
\liminf _{q \rightarrow \infty} \frac{\sum_{i j} P_{i j}\left(\omega \mid L_{n_{q}}(\omega)\right) \log \rho_{i j}\left(n_{q}\right) / \rho_{i}\left(n_{q}\right)}{\sum_{i j} P_{i j}\left(\omega \mid L_{n_{q}}(\omega)\right) \log a_{i j}}+\frac{\sum_{i} P_{i}\left(\omega \mid n_{k}\right) \log \rho_{i}\left(n_{q}\right)}{\sum_{i} P_{i}\left(\omega \mid n_{q}\right) \log b_{i}} \\
=\liminf _{q \rightarrow \infty} \frac{\sum_{i j} P_{i j}\left(\omega \mid L_{n_{q}}(\omega)\right) \log P_{i j}\left(\omega \mid L_{n_{q}}(\omega)\right) / P_{i}\left(\omega \mid L_{n_{q}}(\omega)\right)}{\sum_{i j} P_{i j}\left(\omega \mid L_{n_{q}}(\omega)\right) \log a_{i j}}+\frac{\sum_{i} P_{i}\left(\omega \mid n_{q}\right) \log P_{i}\left(\omega \mid n_{q}\right)}{\sum_{i} P_{i}\left(\omega \mid n_{q}\right) \log b_{i}} .
\end{array}
$$


By the first condition on $n_{q}$ we have

$$
\begin{array}{r}
\liminf _{q \rightarrow \infty} \frac{\sum_{i j} P_{i j}\left(\omega \mid L_{n_{q}}(\omega)\right) \log P_{i j}\left(\omega \mid L_{n_{q}}(\omega)\right) / P_{i}\left(\omega \mid L_{n_{q}}(\omega)\right)}{\sum_{i j} P_{i j}\left(\omega \mid L_{n_{q}}(\omega)\right) \log a_{i j}} \\
+\frac{\sum_{i} P_{i}\left(\omega \mid n_{q}\right) \log P_{i}\left(\omega \mid n_{q}\right)}{\sum_{i} P_{i}\left(\omega \mid n_{q}\right) \log b_{i}} \\
\leq \liminf _{q \rightarrow \infty} \frac{\sum_{i j} P_{i j}\left(\omega \mid L_{n_{q}}(\omega)\right) \log P_{i j}\left(\omega \mid L_{n_{q}}(\omega)\right) / P_{i}\left(\omega \mid L_{n_{q}}(\omega)\right)}{\sum_{i j} P_{i j}\left(\omega \mid L_{n_{q}}(\omega)\right) \log a_{i j}} \\
+\frac{\sum_{i} P_{i}\left(\omega \mid L_{n_{q}}(\omega)\right) \log P_{i}\left(\omega \mid L_{n_{q}}(\omega)\right)}{\sum_{i} P_{i}\left(\omega \mid L_{n_{q}}(\omega)\right) \log b_{i}} .
\end{array}
$$

Since $\lim _{q \rightarrow \infty} \mathbf{P}\left(\omega \mid L_{n_{q}}(\omega)\right)=\mathbf{P}$ and $\mathbf{P} \in A$ we have

$$
\begin{array}{r}
\lim _{q \rightarrow \infty} \frac{\sum_{i j} P_{i j}\left(\omega \mid L_{n_{q}}(\omega)\right) \log P_{i j}\left(\omega \mid L_{n_{q}}(\omega)\right) / P_{i}\left(\omega \mid L_{n_{q}}(\omega)\right)}{\sum_{i j} P_{i j}\left(\omega \mid L_{n_{q}}(\omega)\right) \log a_{i j}} \\
+\frac{\sum_{i} P_{i}\left(\omega \mid L_{n_{q}}(\omega)\right) \log P_{i}\left(\omega \mid L_{n_{q}}(\omega)\right)}{\sum_{i} P_{i}\left(\omega \mid L_{n_{q}}(\omega)\right) \log b_{i}} \\
=\frac{\sum_{i j} P_{i j} \log P_{i j} / P_{i}}{\sum_{i j} P_{i j} \log a_{i j}}+\frac{\sum_{i} P_{i} \log P_{i}}{\sum_{i} P_{i j} \log b_{i}} \leq s .
\end{array}
$$

Hence, for each $\omega \in \Omega$ we may find $\beta(\omega) \in \mathbb{B}$ such that

$$
\liminf _{n \rightarrow \infty} \frac{\log \mu_{\beta(\omega)}\left(B_{n}(\omega)\right)}{\log \prod_{\nu=1}^{n} b_{i_{\nu} j_{\nu}}} \leq s+\delta
$$

Letting $\Lambda^{\Omega}(\beta):=\{x \in \Pi(\Omega): \beta(x)=\beta\}$ for each $\beta \in \mathbb{B}$ we have $\Pi(\Omega)=$ $\bigcup_{\beta \in \mathbb{B}} \Lambda^{\Omega}(\beta)$. Moreover, by 4.9 combined with Lemma 2.3 (ii) we have $\operatorname{dim}_{\mathcal{H}} \Lambda^{\Omega}(\beta) \leq s+\delta$ for each $\beta \in \mathbb{B}$. Since $\operatorname{dim}_{\mathcal{H}}$ is closed under countable unions it follows that $\operatorname{dim}_{\mathcal{H}} \Pi(\Omega) \leq s+\delta$. Letting $\delta \rightarrow 0$ proves the lemma.

We now make a quick digression to see how Lemma 4.2 implies the following generalization of a result due to Nielsen [14]. Given $\mathbf{p}=\left(p_{i j}\right)_{(i, j) \in \mathcal{D}} \in \mathbb{P}$ we define

$$
\Lambda(\mathbf{p}):=\left\{x=\Pi(\omega) \in \Lambda: \lim _{n \rightarrow \infty} P_{i j}(\omega \mid n)=p_{i j} \text { for all }(i, j) \in \mathcal{D}\right\} .
$$

Corollary 1. For each $\mathbf{p} \in \mathbb{P} \operatorname{dim}_{\mathcal{H}}(\Lambda(\mathbf{p}))=D_{L Y}\left(\mu_{\mathbf{p}}\right)$.

Proof. The lower bound follows from several applications of the Kolmogorov's strong law of large numbers combined with Lemma 2.3 (ii). The upper bound is an immediate consequence of Lemma 4.2 with $A=\{\mathbf{p}\}$.

Returning to the proof of Theorem 1 we obtain our first upper estimate for $\operatorname{dim}_{\mathcal{H}} \Lambda_{\alpha}^{\varphi}$. 


\section{Lemma 4.3.}

$$
\operatorname{dim}_{\mathcal{H}} \Lambda_{\alpha}^{\varphi} \leq \sup \left\{D_{L Y}(\mu): \mu \in \mathcal{B}_{\sigma}(\Sigma),\left|\int \varphi d \mu-\alpha\right| \leq \operatorname{var}_{1}(\varphi)\right\} .
$$

Proof. By Lemma 4.2 it suffices to show that given $\omega \in \Sigma_{\alpha}^{\varphi}$ and $\mathbf{p}=\left(p_{i j}\right) \in \mathbb{P}$ a limit point for the sequence $(\mathbf{P}(\omega \mid n))_{n \in \mathbb{N}}$ we have $\left|\int \varphi d \mu_{\mathbf{p}}-\alpha\right| \leq \operatorname{var}_{1}(\varphi)$. Now given $(i, j) \in \mathcal{D}$ we have $\left|\int_{[(i, j)]} \varphi d \mu_{\mathbf{p}}-\varphi(\tau)\right| \leq \operatorname{var}_{1}(\varphi)$ for all $\tau \in \Sigma$ with $\tau_{1}=(i, j)$. Thus, for all $n \in \mathbb{N}$ we have

$$
\left|\sum_{(i, j) \in \mathcal{D}} P_{i j}(\omega \mid n) \int_{[(i, j)]} \varphi d \mu_{\mathbf{p}}-\frac{1}{n} \sum_{l=0}^{n-1} \varphi\left(\omega^{l}\right)\right| \leq \operatorname{var}_{1}(\varphi) .
$$

Since $\mathbf{p}$ is a limit point of $(\mathbf{P}(\omega \mid n))_{n \in \mathbb{N}}$ and $\omega \in \Sigma_{\alpha}^{\varphi}$ this implies

$$
\left|\sum_{(i, j) \in \mathcal{D}} p_{i j} \int_{[(i, j)]} \varphi d \mu_{\mathbf{p}}-\alpha\right| \leq \operatorname{var}_{1}(\varphi) \text {. }
$$

Since $\int \varphi d \mu_{\mathbf{p}}=\sum_{(i, j) \in \mathcal{D}} p_{i j} \int_{[(i, j)]} \varphi d \mu_{\mathbf{p}}$ this completes the proof of the lemma.

Lemma 4.3 proves the special case of Theorem 1 for which $\operatorname{var}_{1}(\varphi)=$ 0 . To prove the upper bound in Theorem 1 in full generality requires a little more work. We iterate our Lalley-Gatzouras system many times to form new Lalley-Gatzouras systems to which we apply Lemma 4.3 to obtain increasingly precise estimates for the upper bound. Take $k \in \mathbb{N}$. For each finite string $\xi:=\xi_{1} \cdots \xi_{k} \in \mathcal{D}^{k}$ we let

$$
S_{\xi}:=S_{\xi_{1}} \circ \cdots \circ S_{\xi_{k}} .
$$

It follows from the fact that $\left(S_{i j}\right)_{(i, j) \in \mathcal{D}}$ is a Lalley-Gatzouras system that $\left(S_{\xi}\right)_{\xi \in \mathcal{D}^{k}}$ is also a Lalley-Gatzouras system, which we call the $k$-th level Lalley-Gatzouras system. $\Sigma$ may be identified with the full shift $\left(\mathcal{D}^{k}\right)^{\mathbb{N}}$. The corresponding left shift is then just $k$ times the ordinary left shift, $\sigma^{k}$. Thus, in order to relate the $k$-th level Lalley-Gatzouras system back to our original Lalley-Gatzouras system we will require a lemma relating members of $\mathcal{M}_{\sigma^{k}}(\Sigma)$ to members of $\mathcal{M}_{\sigma}(\Sigma)$. Define a potential $A_{k}(\varphi): \Sigma \rightarrow \mathbb{R}$ by $A_{k}(\varphi):=\frac{1}{k} \sum_{l=0}^{k-1} \varphi \circ \sigma^{l}$ and for each $\nu \in \mathcal{M}_{\sigma^{k}}(\Sigma)$ define a Borel probability measure $A_{k}(\nu)$ by $A_{k}(\nu):=\frac{1}{k} \sum_{l=0}^{k-1} \nu \circ \sigma^{-l}$. Similarly if $\nu \in \mathcal{M}_{\sigma_{v}^{k}}\left(\Sigma_{v}\right)$ we let $A_{k}^{v}(\nu):=\frac{1}{k} \sum_{l=0}^{k-1} \nu \circ \sigma_{v}^{-l}$.

Lemma 4.4. Take $\nu \in \mathcal{M}_{\sigma^{k}}(\Sigma)$ and let $\mu=A_{k}(\nu)$. Then,

(i) $\mu \in \mathcal{M}_{\sigma}(\Sigma)$

(ii) $h(\mu, \sigma)=\frac{1}{k} h\left(\nu, \sigma^{k}\right)$

(iii) $h^{v}(\mu, \sigma)=\frac{1}{k} h^{v}\left(\nu, \sigma^{k}\right)$ 
(iv) $\lambda(\mu, \sigma)=\frac{1}{k} \lambda\left(\nu, \sigma^{k}\right)$

(v) $\lambda^{v}(\mu, \sigma)=\frac{1}{k} \lambda^{v}\left(\nu, \sigma^{k}\right)$

(vi) $\int \varphi d \mu=\int A_{k}(\varphi) d \nu$

(vii) $D_{L Y}(\mu)=D_{L Y}^{k}(\nu)$.

Proof. Parts (i), (i), (iv), (v) and (vi) follow from [10] Lemma 2. Since $\pi \circ \sigma=\sigma_{v} \circ \pi$ we have $A_{k}^{v}(\pi(\nu))=\pi\left(A_{k}(\nu)\right)$ and hence (iii) also follows from [10] Lemma 2. Part (vii) follows from parts (i), (ii), (iii), (iv) and (v).

\section{Lemma 4.5.}

$$
\operatorname{dim}_{\mathcal{H}} \Lambda_{\alpha}^{\varphi} \leq \sup \left\{D_{L Y}(\mu): \mu \in \mathcal{B}_{\sigma}(\Sigma),\left|\int \varphi d \mu-\alpha\right| \leq \operatorname{var}_{k}\left(A_{k}(\varphi)\right)\right\} .
$$

Proof. First note that $\frac{1}{n} \sum_{l=0}^{n-1} A_{k}(\varphi) \circ\left(\sigma^{k}\right)^{l}=\frac{1}{n k} \sum_{l=0}^{n k-1} \varphi \circ \sigma^{l}$ and hence $\lim _{n \rightarrow \infty} \frac{1}{n} \sum_{l=0}^{n-1} A_{k}(\varphi)\left(\left(\sigma^{k}\right)^{l}(\omega)\right)=\alpha$ for all $\omega \in \Sigma_{\alpha}^{\varphi}$. Thus, by applying Lemma 4.3 to our $k$-th level Lalley-Gatzouras system and noting that words of length $k$ and $\sigma^{k}$ invariant measures in the original system correspond, respectively, to digits and shift invariant measures in the $k$-th level LalleyGatzouras system we have

$$
\operatorname{dim}_{\mathcal{H}} \Lambda_{\alpha}^{\varphi} \leq \sup \left\{D_{L Y}^{k}(\nu): \nu \in \mathcal{B}_{\sigma^{k}}(\Sigma),\left|\int A_{k}(\varphi) d \nu-\alpha\right| \leq \operatorname{var}_{k}\left(A_{k}(\varphi)\right)\right\} .
$$

Combining 4.14 with Lemma 4.4 proves the lemma.

Since $\varphi \in C(\Sigma)$ is continuous we have $\lim _{k \rightarrow \infty} \operatorname{var}_{k}\left(A_{k}(\varphi)\right)=0$. Thus, by Lemma 4.5 , we have shown

$$
\operatorname{dim}_{\mathcal{H}} \Lambda_{\alpha}^{\varphi} \leq \sup \left\{D_{L Y}(\mu): \mu \in \mathcal{M}_{\sigma}(\Sigma),\left|\int \varphi d \mu-\alpha\right| \leq \epsilon\right\}
$$

for arbitrarily small $\epsilon>0$ and so by Lemma 4.1 the result follows.

\section{REMARKS}

Following Olsen and Winter [15], [16] one may consider more general types of level sets. Given $A \subseteq\left[\alpha_{\min }(\varphi), \alpha_{\max }(\varphi)\right]$ we let $\overline{\Sigma_{A}^{\varphi}}$ denote the set of $\omega \in \Sigma$ for which every accumulation point of the sequence $\left(A_{n}(\varphi)(\omega)\right)_{n \in \mathbb{N}}$ lies within $A$ and $\overline{\Lambda_{A}^{\varphi}}:=\Pi\left(\overline{\Sigma_{A}^{\varphi}}\right)$ its projection by $\Pi$. Then, by essentially the same argument as above, we have

$$
\operatorname{dim}_{\mathcal{H}} \overline{\Lambda_{A}^{\varphi}}=\sup \left\{D_{L Y}(\mu): \mu \in \mathcal{M}_{\sigma}(\Sigma), \int \varphi d \mu \in A\right\} .
$$

Now suppose $A \subseteq\left[\alpha_{\min }(\varphi), \alpha_{\max }(\varphi)\right]$ is a compact sub-interval. Let $\Sigma_{A}^{\varphi}$ denote the set of $\omega \in \Sigma$ for which the set of accumulation points of the sequence $\left(A_{n}(\varphi)(\omega)\right)_{n \in \mathbb{N}}$ is equal to $A$ and $\Lambda_{A}^{\varphi}:=\Pi\left(\Sigma_{A}^{\varphi}\right)$. By employing the 
methods of [16] Theorem 3.1, along with a few ideas from section 3 , one can prove the following lower estimate

$$
\operatorname{dim}_{\mathcal{H}} \underline{\Lambda_{A}^{\varphi}} \geq \inf _{\alpha \in A} \sup \left\{D_{L Y}(\mu): \mu \in \mathcal{M}_{\sigma}(\Sigma), \int \varphi d \mu=\alpha\right\} .
$$

In particular, the projection of the set of points $\omega \in \Sigma$ for which $\left(A_{n}(\varphi)(\omega)\right)_{n \in \mathbb{N}}$ does not converge has dimension $\operatorname{dim}_{\mathcal{H}} \Lambda$. However, it seems very plausible that the lower bound given by $(5.2)$ is not always optimal and it would be interesting to know what the exact value of $\operatorname{dim}_{\mathcal{H}} \underline{\Lambda_{A}^{\varphi}}$ is.

\section{REFERENCES}

[1] J. Barral and D. Feng, Weighted thermodynamic formalism and applications, (2009). arXiv:0909.4247v1.

[2] J. Barral and M. Mensi, Multifractal analysis of Birkhoff averages on 'self-affine' symbolic spaces. Nonlinearity 21 (2008), no. 10, 2409-2425.

[3] K. Barański, Hausdorff dimension of the limit sets of some planar geometric constructions Adv. Math. 210.1 (2007), 391-415.

[4] L. Barriera and B. Saussol, Variational principles and mixed multifractal spectra. Trans. Amer. Math. Soc. 353 (2001), no. 10, 3919-3944.

[5] T. Bedford, PhD Thesis: Crinkly curves, Markov partitions and box dimension of self-similar sets. Ph.D. thesis, University of Warwick (1984).

[6] K. Falconer, Techniques in Fractal Geometry. John Wiley and Sons, Ltd., Chichester, (1997).

[7] A. Fan, D. Feng and J. Wu, Recurrence, dimension and entropy. J. London Math. Soc. (2) 64 (2001), no. 1, 229-244.

[8] S. P. Lalley and D. Gatzouras, Hausdorff and Box Dimension of certain Self-Affine Fractals, Indiana Univ. Math. J. 41 (1992), 533.

[9] K. Gelfert and M. Rams, The Lyapunov spectrum of some parabolic systems. Ergodic Theory Dynam. Systems 29 (2009), no. 3, 919-940.

[10] A. Johansson, T. Jordan, A. Oberg, and M. Pollicott, Multifractal analysis of nonuniformly hyperbolic systems. Isreal J. Math., Vol 177, 125-144, (2008).

[11] T. Jordan and K. Simon, Multifractal Analysis of Birkhoff Averages for some SelfAffine IFS. Dynamical Systems, Vol 22, Issue 4, (2007), 469-483.

[12] F. Ledrappier and L. S. Young, The Metric Entropy of Diffeomorphisms: Part II: Relations between Entropy, Exponents and Dimension, Ann. of Math. 122 (1985) 509-574.

[13] C. McMullen, The Hausdorff Dimension of General Sierpinski Carpets. Nagoya Maths Journal, vol. 96 (1984).

[14] O. Nielsen, The Hausdorff and packing dimensions of some sets related to Sierpinski carpets. Canad. J. Math. 51 (1999), 1073-1088.

[15] L. Olsen, Multifractal analysis of divergence points of deformed measure theoretical Birkhoff averages. J. Math. Pures Appl. (9) 82 (2003), no. 12, 1591-1649.

[16] L. Olsen and S. Winter, Multifractal analysis of divergence points of deformed measure theoretical Birkhoff averages. II. Non-linearity, divergence points and Banach space valued spectra. Bull. Sci. Math. 131 (2007), no. 6, 518-558.

[17] Y. Pesin, Dimension Theory in Dynamical Systems. Contemporary views and applications. Chicago Lectures in Mathematics. University of Chicago Press, Chicago, IL, (1997).

[18] Y. Pesin and H. Weiss, The Multifractal Analysis of Birkhoff Averages and Large Deviations. Global Analysis of Dynamical Systems, 419-431. Inst. Phys., Bristol, (2001). 
[19] P. Walters, An Introduction to Ergodic Theory. Graduate Texts in Mathematics, 79. Springer-Verlag, New York-Berlin, (1982).

Henry WJ Reeve, Department of Mathematics, The University of Bristol, University Walk, Clifton, Bristol, BS8 1TW, UK

E-mail address: henrywjreeve@googlemail.com 